\title{
The basic science of glomus jugulare tumors
}

\author{
JASON HETH, M.D. \\ Department of Neurosurgery, University of Michigan, Ann Arbor, Michigan
}

\begin{abstract}
Glomus tumors are a fascinating group of lesions. It is a challenge for neurosurgeons and otolaryngologists to resect them completely with minimal morbidity. Laboratory researchers have discovered extremely interesting genetic and molecular biology factors involved in the development and growth of glomus tumors. In this article the author reviews the genetics, protein mutations, angiogenesis and apoptosis associated with tumor formation, and the secretion of vasoactive substances is discussed as well. It is hoped that with further research less invasive measures may be developed to treat these tumors.
\end{abstract}

\section{KEY WoRDS • glomus jugulare tumor genetic imprinting}

Skull base surgery has made significant strides in its ability to resect skull base tumors. Advanced instrumentation, surgical procedures, anesthetic agents, and reconstructive techniques have made this possible. Several tumors continue to present challenges despite their benign biological character; glomus tumors fit this description. Glomus jugulare tumors are fascinating lesions that arise from paraganglia cells situated near the jugular foramen. Their intimate association with the lower cranial nerves and inner ear complicates their management, because injuries to these structures can cause significant morbidity and functional disability. Knowledge of the basic biology of glomus tumors may result in improved therapies or a molecular-level noninvasive treatment. Basic research in glomus tumors has surged and subsequent advances in understanding of the basic biology of glomus jugulare tumors have been exciting. This review examines the basic epidemiological features of glomus tumors that led to further inquiry. Such investigations have resulted in important advances in the understanding of the genetic and molecular biology aspects of glomus tumors. Angiogenesis and apoptosis in tumor formation are examined, and production of vasoactive substances is reviewed.

A preliminary note about terminology is in order because these tumors have been called by many names. Glomus tumors and paragangliomas are two conventions in common use. In keeping with the chosen topic, the tumors of interest will be referred to as glomus jugulare tumors. In many studies all tumor subgroups are pooled to increase the amount of data, and therefore all tumors in this review will be referred to as glomus tumors.

Abbreviations used in this paper: $\mathrm{PD}-\mathrm{ECGF}=$ platelet-derived endothelial cell growth factor; $\mathrm{SDH}=$ succinate dehydrogenase; SDHA, B, C, and D = SDH subunits A, B, C, and D; VEGF = vascular endothelial growth factor.

\section{BASIC SCIENCE}

\section{Epidemiological Features}

Paraganglion tissue is derived from the neural crest: chief cells, the major component of the pathological hallmark zellballen, migrate during embryogenesis from the neural crest to concentrate around the autonomic ganglia. The largest such collection is the adrenal medulla; however, other collections are found near the jugular foramen, the middle ear, the vagal nerve, the carotid bifurcation, and around the aorta. They are thought to store and secrete catecholamines in response to neuronal or chemical signals ${ }^{68}$ so paraganglia act as an endocrine tissue. Additionally, the carotid body acts as an oxygen sensor. Guild ${ }^{26}$ first described the paraganglion tissue found in the middle ear, and Rosenwasser ${ }^{51}$ first found a correlation between a vascular middle ear tumor and the paraganglion tissue described by Guild.

Glomus tumors occur at a rate of approximately one case per 30,000 individuals ${ }^{37}$ and represent $0.012 \%$ of all tumors diagnosed in humans. ${ }^{34}$ Greer and colleagues ${ }^{24}$ reported an incidence of 8.6 cases per 100,000 new patients. Glomus jugulare tumors and glomus tympanicum lesions are the second most common temporal bone tumor. Female patients are affected approximately two to five times more frequently than are male patients. ${ }^{1,24,57}$ Nonfamilial tumors are multicentric in $10 \%$ of cases. ${ }^{8,56}$ Familial glomus tumors constitute approximately $20 \%$ of lesions found in patients with glomus tumors, and in these individuals multicentricity occurs in approximately 25 to $78 \%$ of cases. ${ }^{29,41,63}$ Van der Mey and colleagues ${ }^{63}$ examined the $^{2}$ pedigrees of 15 families in which multiple family members had at least one glomus tumor. In children of men who had a glomus tumor, these lesions developed at a rate consistent with autosomal-dominant inheritance (50\%). The children of women with glomus tumors did not have 
this type of lesion; nevertheless, these tumors could arise in members of subsequent generations as long as the affected parent was the father. That is to say, a man whose mother had a familial glomus tumor would not have a glomus tumor, but tumors could develop in his children in an autosomal-dominant fashion. This is an example of genomic imprinting: in some fashion, the responsible gene from an affected mother is inactivated but can be reactivated later if one of her sons inherits the altered gene. This interesting finding led researchers to the fascinating process with which they elucidated the genetic and molecular basis for familial glomus tumors.

\section{Genetic and Molecular Basis of Glomus Tumors}

Genetic linkage analysis was undertaken to identify the gene or genes responsible for familial glomus tumors. With such analysis, researchers first identified two putative regions on chromosome 11: PGL1 at 11q23.127,28 and PGL2 at 11q13.1. ${ }^{38}$ Both of these loci were associated with maternal imprinting. ${ }^{16,58}$ Baysal, et al. ${ }^{6}$ first identified germline mutations in $S D H D$ at the $P G L 1$ location. The $S D H D$ gene encodes for a protein called alternately succinate-ubiquinone reductase subunit D or SDHD. The protein product for PGL2 has not yet been elucidated. More families were described in which either genomic imprinting was not found or the PGL1 and PGL2 loci were excluded as harboring the genetic mutations. Niemann and colleagues ${ }^{43,44}$ described the PGL3 locus on chromosome 1q. The gene was found to encode SDHC. ${ }^{43}$ Mutations at another locus (PGL4) on chromosome 1p36 cause mutations in SDHB. Neither of these genes displays genetic imprinting. ${ }^{4,43}$

These genes encode for portions of proteins called alternately SDH or succinate-ubiquinone oxidoreductase, which contribute to mitochondrial complex II. The subunits encoded by $P G L$ loci are SDHD, SDHC, and SDHB; SDHC and SDHD are membrane-bound proteins that anchor SDHA and SDHB. Incidentally, mutations in SDHA cause a fatal and unrelated juvenile encephalopathy called Leigh disease. ${ }^{10}$ The SDH is localized to the inner mitochondrial membrane, where both the Krebs cycle and the mitochondrial respiratory chain contain the reaction it catalyzes. Within the Krebs cycle, it converts succinate to fumarate, reducing one molecule of flavin adenine dinucleotide to $\mathrm{FADH}_{2} \cdot{ }^{36}$ Within the respiratory chain it catalyzes the conversion of ubiquinone to dihydroubiquinone by using flavin adenine dinudeotide as an intermediary. The dihydroubiquinone continues as an electron carrier in the mitochondrial electron transport chain. The molecular cascade between SDH mutations and tumor formation is unknown and remains an area of active investigation.

Baysal, et al., ${ }^{6}$ proposed that SDHD is a critical component of a cellular oxygen-sensing system. Mutations in SDHD may incapacitate the oxygen-sensing mechanism, leading to an apparent or real hypoxic state accompanied by chronic hypoxic stimulation and cell proliferation. Support for hypoxia-induced hyperplasia comes from evidence obtained in high-altitude physiological studies. Cows, guinea pigs, rabbits, and dogs experience carotid body hyperplasia when living at high altitudes, which exposes them to a hypoxic condition; ${ }^{2,17}$ this has also been described in humans. ${ }^{3}$ In people living at higher altitudes, glomus jugulare tumors and glomus tumors also develop at higher rates compared with rates in people living at sea level, ${ }^{52}$ with a female/male ratio of 8.3:1. ${ }^{50}$ Another clinical observation lending support to these theories is the finding that patients suffering from conditions resulting in hypoxemia, such as cystic fibrosis, cyanotic heart disease, and chronic obstructive pulmonary disease experience carotid body hyperplasia, ${ }^{33,35}$ and those suffering from chronic obstructive pulmonary disease have a higher rate of carotid body tumors. ${ }^{52}$ This relationship between SDHD, SDHC, and SDHB mutations and the oxygensensing mechanism, and the cascade of events between these mutations and tumor formation are not completely known. These areas will continue to be actively investigated.

Neurofibromatosis is associated with both pheochromocytomas and glomus tumors. ${ }^{14}$ Pheochromocytomas are also associated with multiple endocrine neoplasia Type II with RET gene mutations, and with von Hippel-Lindau syndrome and mutations in the VHL gene. ${ }^{42}$ Carney, et al., ${ }^{11}$ have suggested a syndrome consisting of a triad of gastric leiomyosarcoma, functioning extraadrenal glomus tumor and pulmonary chondroma, ${ }^{11}$ although no studies have demonstrated a genetic defect.

It is also interesting to note that van der Mey, et al., ${ }^{63}$ found that in patients in successive generations of a family harboring the mutations, tumors developed at progressively younger ages. This finding is a good example of genetic anticipation, in which the mutation appears to be more severe with succeeding generations.

In summary, important aspects of glomus tumor genetics have been uncovered. Many questions remain about the PGL2 locus, the SDH mutations, the nature of the oxygen sensor, and the steps between mutation and tumor formation. The outlook is promising that these questions will be answered in the future.

\section{Molecular Correlations in Glomus Jugulare Tumor Formation}

Familial glomus tumors constitute approximately $20 \%$ of lesions, for which the genetic defects are known. One subgroup $(<10 \%$ of all glomus tumors) appears to be caused by sporadic mutations in SDHB and SDHD., ${ }^{7,21}$ The pathogenesis of the remaining $80 \%$ of tumors remains a mystery. Oncology research implicates several processes as important to tumor formation and growth. Two that attract a significant amount of attention are angiogenesis and apoptosis, and these processes have been examined in glomus jugulare tumors as well. Given the rarity of glomus jugulare tumors and the similarity of these lesions to other glomus tumors, all data from glomus tumors are frequently pooled to increase the material from which to draw conclusions. In some studies pheochromocytomas may be pooled with glomus tumors of the head and neck. Conclusions drawn from these studies may be generalizable to glomus jugulare tumors as a subgroup; however, their validation in glomus jugulare tumors would be preferable.

Angiogenesis is a critical event in the development and sustained growth of several tumor types. Given the vascular nature of paraganglion tissue, questions about the role 
that angiogenic molecules play in the development of glomus jugulare tumors naturally arise. Jyung, et al., ${ }^{30}$ examined 17 paraganglioma specimens and found VEGF in 11 $(65 \%)$ of 17 and PD-ECGF in $10(77 \%)$ of 13 when looking at chief cells, whereas PD-ECGF was found in the fibrovascular stroma. Basic fibroblast growth factor, platelet-derived growth factor, transforming growth factor- $\beta 1$, and angiogenin (other angiogenic growth factors) were not found. These authors suggested that VEGF and PDECGF may play a critical role in glomus tumor development. Others also found VEGF by performing immunohistochemical analysis in extraadrenal glomus tumor tissue. ${ }^{53}$ Watanabe, et al. ${ }^{69}$ found eight (73\%) of 11 glomus tumors positive for endothelin-1 and 72 to $73 \%$ positive for endothelin receptors. They suggested that this was consistent with a paracrine mechanism for tumor development. Analysis of the data indicates that VEGF and the endothelins and their receptors may play a role in glomus jugulare tumor development.

Cohorts frequently included patients with pheochromocytomas, whereas literature looking at angiogenesis in vitro by using immortalized rat PC12 pheochromocytoma cells also increased. The VEGF and its receptors have been consistently implicated in pheochromocytoma development. ${ }^{19,53,60,70}$ In experiments in which PC12 cells were injected subcutaneously in rats, ingrowth of endothelial cells was found, ${ }^{40}$ and treatment with an anti-VEGF antibody reduced tumor size and vascularization. ${ }^{70}$ Mutations in SDHB and SDHD genes cause complete loss of electron transport chain complex II activity in mitochondria in pheochromocytomas as well as upregulation of VEGF and its receptors. ${ }^{21,22}$ These data all indicate an important role for VEGF in pheochromocytoma development.

The endothelin system has also been found in pheochromocytomas: endothelin-1 has been found in 19 (59\%) of 32 pheochromocytomas, whereas endothelin receptors have been found in 66 to $78 \%$ of tumors. ${ }^{69}$ The endothelin receptor Type B has been found in vascular endothelial cells, ${ }^{19}$ and endothelin-1 stimulates production of tyrosine hydroxylase and dopamine in PC12 cells. ${ }^{59}$ Although pheochromocytomas are a glomus tumor, PC12 cells may differ significantly from glomus jugulare tumors cells, not only because of their location, their origin in rats, and different vasoactive substance production, but also because the immortalization and passaging processes alter the cells' behavior over time. Therefore, caution must be exercised when interpreting data obtained in PC12 cells and attempting to extrapolate it to glomus jugulare tumors.

Not surprisingly, hypoxia induces expression of hypoxia inducible factor $1 \alpha$, which participates in the angiogenesis cascade. Histological sections of pheochromocytomas exhibit ubiquitous hypoxia-inducible factor $1 \alpha$ staining. ${ }^{19}$ Because the mutations result in cell hypoxia, this may represent a link between the mutation and tumor development.

Apoptosis is the process of programmed cell death. It occurs during organism development to control growth patterns during the transition from fetus to adult. It also occurs as a cellular mechanism to eliminate tissue that is developing abnormally. The proapoptotic proteins p53 and bax are critical in facilitating this process; cell mutations in p53 can increase abnormal cell survival. On the other hand, bcl-2 is an antiapoptotic homolog of bax: overexpression of bcl-2 forestalls apoptosis and increases cell survival. Data on these proteins in glomus jugulare tumors are limited. Pavai and colleagues ${ }^{45}$ examined paraganglioma specimens, including 12 glomus jugulare tumors, by using immunohistochemistry to study pathological specimens. They found nine of 23 tumors and none of the four glomus jugulare tumors to be positive for bcl-2. They found the apoptotic protein bax in 26 of 28 tumors and in all five glomus jugulare tumors. They concluded that the low rate of bcl-2 positivity and the higher rate of bax positivity may have resulted in the generally indolent behavior exhibited by glomus tumors.

In other reports apoptosis is investigated in head and neck glomus tumors as a group. In several of these reports high levels of bcl-2 expression were found, in contrast to the findings of Pavai and colleagues. Wang, et al.. ${ }^{66}$ found 11 of 13 carotid body tumors positive for bcl-2 on immunohistochemical studies. They proposed that the presence of bcl-2 contributes to the indolent course of glomus tumors. Dekker, et al. ${ }^{15}$ found bcl-x 1 positivity in 40 of 41 cases of head and neck paragangliomas. They found that p53 immunohistochemical staining was either absent or very faint. They used terminal deoxynucleotidyl transferase-mediated deoxyuridine triphosphate nick-end labeling to examine tumor sections for apoptotic cell death, yielding "only occasional apoptotic bodies." They concluded there is no significant apoptotic activity in glomus tumors. Van Nederveen, et al., ${ }^{64}$ found p53 immunoreactivity in 15 of 43 tumors; three of 43 had p53 and MDM2 positivity (MDM2 targets p53 for destruction and forestalls apoptosis), and 28 of 43 tumors lacked both p53 and MDM2. These authors found no correlation between SDHD mutation status and $\mathrm{p} 53$ expression. They also concluded that p53 mutations do not contribute to paraganglioma tumor formation.

In other studies in which pheochromocytomas were investigated, in either pathological specimens or in PC12 cells, low rates of $\mathrm{p} 53$ positivity, ${ }^{12}$ high rates of bcl-2 positivity, ${ }^{31,67}$ and high rates of bax positivity were also found. ${ }^{31}$ The presence of bcl-2 has been thought to prolong cell survival in pheochromocytomas by blocking proapoptotic agents $\mathrm{s}^{20,67}$ or by slowing cell death while other oncogenes are activated. ${ }^{12,65,67} \mathrm{An}$ inducible transcription factor, NF- $\kappa B$, may also play a role in apoptotic cell death, but that role is still under active investigation. ${ }^{18}$

Overall, the data on apoptosis in glomus jugulare tumors is limited, and a variety of tumor types is represented. With the exception of the report by Pavai, et al., ${ }^{45}$ the bulk of the data indicates that abnormalities in the apoptotic cascade do not contribute to the development of glomus jugulare tumors. The exception to this may be the apoptotic delay caused by bcl-2 that could widen the window of opportunity for activation of other oncogenes.

Telomerase is an enzyme that adds nucleotides to chromosome ends, or telomeres. As an organism ages, telomerase activity dissipates. Consequently, nucleotides that are lost from the telomeres are not replaced, and this is thought to be a molecular representation of senescence. Processes that inhibit telomerase activity accelerate cell death, and those that increase its activity promote cell survival. Data are mixed with regard to involvement of 
telomerase in pheochromocytoma development, with one study demonstrating that the rate of telomerase reversetranscriptase positivity in pathological specimens is the same as in adjacent normal adrenal tissue ${ }^{31}$ and another hypothesizing that bcl-2 and telomerase act to prolong cell survival during tumor development. ${ }^{20}$

\section{Production of Vasoactive Amines}

Glomus jugulare tumors secreting catecholamines occur symptomatically in 1 to $3 \%$ of cases. ${ }^{41,55}$ Glomus tumors have long been known to produce vasoactive substances. In one early classification, glomus tumors were subdivided into chromaffin and nonchromaffin subgroups. The chromaffin staining procedure revealed vasoactive amines, and therefore this subdivision was thought to differentiate vasoactive tumors from nonproductive ones. The chromaffin staining procedure proved to be inconsistent and lacking sensitivity,,$^{13,23,25}$ and thus this categorization and staining procedure fell out of favor in the histological evaluation of glomus tumors.

The vasoactive and endocrine nature of these tumors qualified glomus jugulare tumors as amine precursor uptake and decarboxylation system tumors or APUDomas. ${ }^{46,47}$ Cells included in this series included pituitary corticotrophs, pancreatic islet cells, and carotid body cells. The concept and designation was updated by Pearse ${ }^{48}$ in 1984 as the diffuse neuroendocrine system.

The catecholamine synthetic pathway is shown in Fig. 1. In glomus jugulare tumors, chief cells take up tyrosine and convert it by sequential steps to norepinephrine or epinephrine. The only paraganglion tissue that produces phenylethanolamine-N-methyltransferase in healthy

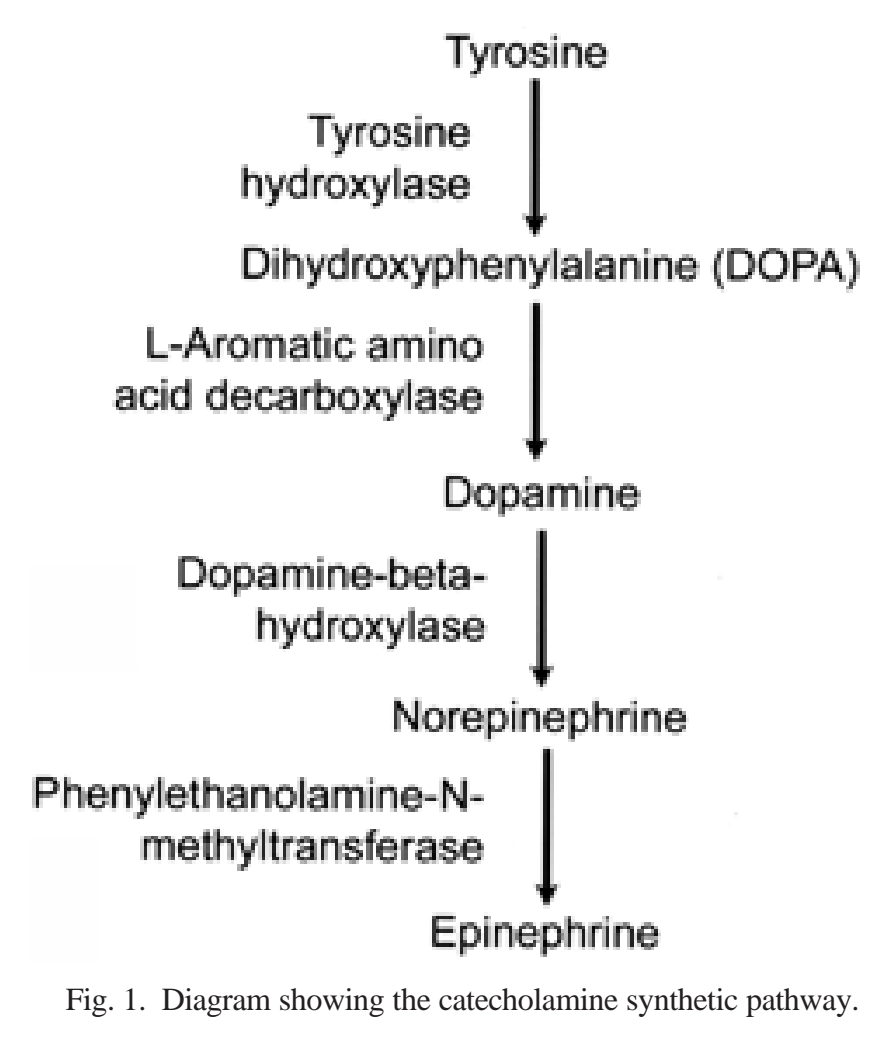

adults is the adrenal medulla. ${ }^{39}$ Consequently, the glomus tumor that most frequently produces epinephrine is the pheochromocytoma. Similarly, functional glomus jugulare tumors and head and neck glomus tumors typically produce norepinephrine. Nevertheless, examples of epinephrine- and phenylethanolamine-N-methyltransferaseproducing glomus jugulare tumors ${ }^{8,9}$ and dopamine- producing glomus jugulare tumors ${ }^{5,55,62}$ have been reported.

The catecholamine degradation pathway is shown in Fig. 2. This pathway is clinically important because the breakdown products metanephrine, normetanephrine, and vanillylmandelic acid can be measured in serum and urine. Elevations to four or five times the upper limit of normal are required to cause clinical symptoms..$^{55}$ Patients with breakdown products measured above this threshold should undergo $\alpha$ - and $\beta$-adrenergic receptor pharmacological blockade before surgery, because impressive blood pressure fluctuations may occur intraoperatively in symptomatic patients without blockade.

Somatostatin is a small-peptide hormone with a multitude of actions in many tissues throughout the body that are mediated by somatostatin receptors. Glomus tumors display somatostatin receptors at a high rate in vitro. ${ }^{32,49}$ This phenomenon has been harnessed clinically as the basis for somatostatin receptor scintigraphy, in which radiolabeled octreotide, a somatostatin analog, is used. This method can be used for postoperative follow up or to search for additional tumor locations. ${ }^{54,61}$

\section{CONCLUSIONS}

Glomus jugulare tumors are a member of the fascinating glomus tumor family. These generally benign lesions are a challenge to resect completely while avoiding cranial nerve morbidity. Although modern microsurgical techniques have enabled good progress toward these goals, a noninvasive method to eradicate glomus jugulare tumors would certainly be welcome. The genetic and molecular biological understanding of these tumors has expanded significantly in the past decade. Concomitantly, the nature of the angiogenic process during tumor development has been actively investigated. Continued research in these areas may yield molecular-level therapies to treat glomus jugulare tumors. This would be a critical step forward in their treatment.

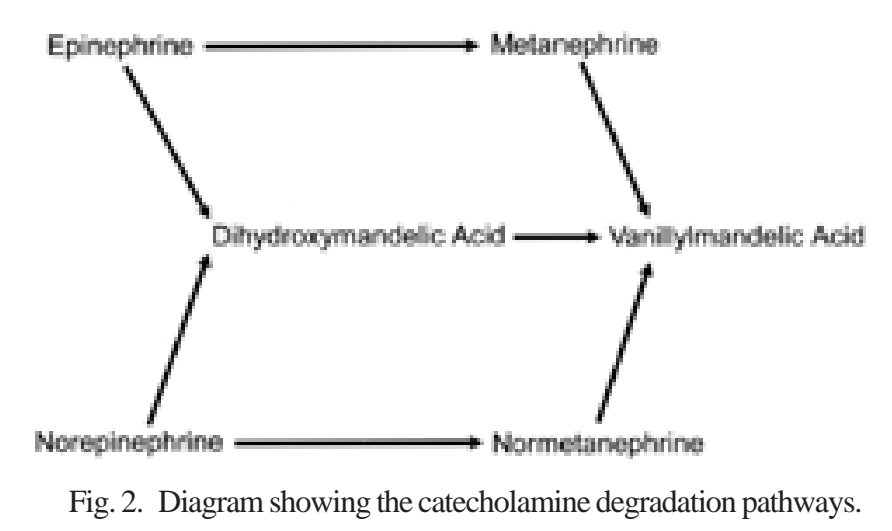




\section{References}

1. Alford BR, Guilford FR: A comprehensive study of tumors of the glomus jugulare. Laryngoscope 72:765-805, 1962

2. Arias-Stella J, Bustos F: Chronic hypoxia and chemodectomas in bovines at high altitudes. Arch Pathol Lab Med 100: 636-639, 1976

3. Arias-Stella J, Valcarcel J: Chief cell hyperplasia in the human carotid body at high altitudes; physiologic and pathologic significance. Hum Pathol 7:361-373, 1976

4. Astuti D, Latif F, Dallol A, et al: Gene mutations in the succinate dehydrogenase subunit SDHB cause susceptibility to familial pheochromocytoma and to familial paraganglioma. Am J Hum Genet 69:49-54, 2001

5. Azzarelli B, Felten S, Muller J, et al: Dopamine in paragangliomas of the glomus jugulare. Laryngoscope 98:573-578, 1988

6. Baysal BE, Ferrell RE, Willett-Brozick JE, et al: Mutations in SDHD, a mitochondrial complex II gene, in hereditary paraganglioma. Science 287:848-851, 2000

7. Baysal BE, Willett-Brozick JE, Lawrence EC, et al: Prevalence of SDHB, SDHC, and SDHD germline mutations in clinic patients with head and neck paragangliomas. J Med Genet 39: 178-183, 2002

8. Blumenfeld J, Cohen N, Anwar M, et al: Hypertension and a tumor of the glomus jugulare region. Evidence for epinephrine biosynthesis. Am J Hypertens 6:382-387, 1993

9. Blumenfeld JD, Cohen N, Laragh JH, et al: Hypertension and catecholamine biosynthesis associated with a glomus jugulare tumor. N Engl J Med 327:894-895, 1992

10. Bourgeron T, Rustin P, Chretien D, et al: Mutation of a nuclear succinate dehydrogenase gene results in mitochondrial respiratory chain deficiency. Nat Genet 11:144-149, 1995

11. Carney JA, Sheps SG, Go VL, et al: The triad of gastric leiomyosarcoma, functioning extra-adrenal paraganglioma and pulmonary chondroma. N Engl J Med 296:1517-1518, 1977

12. de Krijger RR, van der Harst E, van der Ham F, et al: Prognostic value of $\mathrm{p} 53$, bcl-2, and c-erbB-2 protein expression in phaeochromocytomas. J Pathol 188:51-55, 1999

13. De Lellis RA, Roth JA: Norepinephrine in a glomus jugulare tumor. Histochemical demonstration. Arch Pathol 92:73-75, 1971

14. DeAngelis LM, Kelleher MB, Post KD, et al: Multiple paragangliomas in neurofibromatosis: a new neuroendocrine neoplasia. Neurology 37:129-133, 1987

15. Dekker PB, Kuipers-Dijkshoorn N, Hogendoorn PC, et al: G2M arrest, blocked apoptosis, and low growth fraction may explain indolent behavior of head and neck paragangliomas. Hum Pathol 34:690-698, 2003

16. Devilee P, van Schothorst EM, Bardoel AF, et al: Allelotype of head and neck paragangliomas: allelic imbalance is confined to the long arm of chromosome 11, the site of the predisposing locus PGL. Genes Chromosomes Cancer 11:71-78, 1994

17. Edwards C, Heath D, Harris P, et al: The carotid body in animals at high altitude. J Pathol 104:231-238, 1971

18. Erlandsson N, Baumann B, Rossler OG, et al: Lack of correlation between NF-kappaB activation and induction of programmed cell death in PC12 pheochromocytoma cells treated with 6-hydroxydopamine or the cannabinoid receptor 1-agonist CP55,940. Biochem Pharmacol 64:487-495, 2002

19. Favier J, Plouin PF, Corvol P, et al: Angiogenesis and vascular architecture in pheochromocytomas: distinctive traits in malignant tumors. Am J Pathol 161:1235-1246, 2002

20. Fu W, Begley JG, Killen MW, et al: Anti-apoptotic role of telomerase in pheochromocytoma cells. J Biol Chem 274: 7264-7271, 1999

21. Gimenez-Roqueplo AP, Favier J, Rustin P, et al: Functional consequences of a SDHB gene mutation in an apparently sporadic pheochromocytoma. J Clin Endocrinol Metab 87: 4771-4774, 2002
22. Gimenez-Roqueplo AP, Favier J, Rustin P, et al: The R22X mutation of the SDHD gene in hereditary paraganglioma abolishes the enzymatic activity of complex II in the mitochondrial respiratory chain and activates the hypoxia pathway. Am J Hum Genet 69:1186-1197, 2001

23. Glenner GG, Crout JR, Roberts WC: A functional carotid-bodylike tumor. Secreting levarterenol. Arch Pathol 73:230-240, 1962

24. Greer JA, Cody TR, Weiland LH: Neoplasms of the temporal bone. J Otolaryngol 5:391-398, 1976

25. Grimley PM, Glenner GG: Histology and ultrastructure of carotid body paragangliomas. Comparison with the normal gland. Cancer 20:1473-1488, 1967

26. Guild SR: A hitherto unrecognized structure, the glomus jugularis, in man. Am Assoc Anat 79 (Suppl 1):28, 1941 (Abstract)

27. Heutink P, van der Mey AG, Sandkuijl LA, et al: A gene subject to genomic imprinting and responsible for hereditary paragangliomas maps to chromosome 11q23-qter. Hum Mol Genet 1:7-10, 1992

28. Heutink P, van Schothorst EM, van der Mey AG, et al: Further localization of the gene for hereditary paragangliomas and evidence for linkage in unrelated families. Eur J Hum Genet 2: $148-158,1994$

29. Hodge KM, Byers RM, Peters LJ: Paragangliomas of the head and neck. Arch Otolaryngol Head Neck Surg 114:872-877, 1988

30. Jyung RW, LeClair EE, Bernat RA, et al: Expression of angiogenic growth factors in paragangliomas. Laryngoscope 110: 161-167, 2000

31. Kanauchi $\mathrm{H}$, Wada $\mathrm{N}$, Clark $\mathrm{OH}$, et al: Apoptosis regulating genes, bcl-2 and bax, and human telomerase reverse transcriptase messenger RNA expression in adrenal tumors: possible diagnostic and prognostic importance. Surgery 132:1021-1027, 2002

32. Krenning EP, Kwekkeboom DJ, Bakker WH, et al: Somatostatin receptor scintigraphy with [111In-DTPA-D-Phe1]- and [123I-Tyr3]-octreotide: the Rotterdam experience with more than 1000 patients. Eur J Nucl Med 20:716-731, 1993

33. Lack EE: Hyperplasia of vagal and carotid body paraganglia in patients with chronic hypoxemia. Am J Pathol 91:497-516, 1978

34. Lack EE, Cubilla AL, Woodruff JM, et al: Paragangliomas of the head and neck region: a clinical study of 69 patients. Cancer 39:397-409, 1977

35. Lack EE, Perez-Atayde AR, Young JB: Carotid body hyperplasia in cystic fibrosis and cyanotic heart disease. A combined morphometric, ultrastructural, and biochemical study. Am J Pathol 119:301-314, 1985

36. Lodish H, Baltimore D, Berk A, et al: Molecular Cell Biology, ed 3. New York: Scientific American Books, 1995, pp 750, 755, 761-770

37. Mariman EC, van Beersum SE, Cremers CW, et al: Analysis of a second family with hereditary non-chromaffin paragangliomas locates the underlying gene at the proximal region of chromosome 11q. Hum Genet 91:357-361, 1993

38. Mariman EC, van Beersum SE, Cremers CW, et al: Fine mapping of a putatively imprinted gene for familial non-chromaffin paragangliomas to chromosome 11q13.1: evidence for genetic heterogeneity. Hum Genet 95:56-62, 1995

39. McCaffrey TV, Myssiorek D, Marrinan M: Head and neck paragangliomas: physiology and biochemistry. Otolaryngol Clin North Am 34:837-844, v, 2001

40. Middeke M, Hoffmann S, Hassan I, et al: In vitro and in vivo angiogenesis in PC12 pheochromocytoma cells is mediated by vascular endothelial growth factor. Exp Clin Endocrinol Diabetes 110:386-392, 2002

41. Netterville JL, Jackson CG, Miller FR, et al: Vagal paraganglioma: a review of 46 patients treated during a 20 -year period. Arch Otolaryngol Head Neck Surg 124:1133-1140, 1998 
42. Neumann HP, Bausch B, McWhinney SR, et al: Germ-line mutations in nonsyndromic pheochromocytoma. N Engl J Med 346:1459-1466, 2002

43. Niemann S, Becker-Follmann J, Nurnberg G, et al: Assignment of PGL3 to chromosome 1 (q21-q23) in a family with autosomal dominant non-chromaffin paraganglioma. Am J Med Genet 98:32-36, 2001

44. Niemann S, Steinberger D, Muller U: PGL3, a third, not maternally imprinted locus in autosomal dominant paraganglioma. Neurogenetics 2:167-170, 1999

45. Pavai Z, Orosz Z, Horvath E, et al: Immunohistochemical features of paragangliomas. J Cell Mol Med 5:311-316, 2001

46. Pearse AG: Common cytochemical and ultrastructural characteristics of cells producing polypeptide hormones (the APUD series) and their relevance to thyroid and ultimobranchial C cells and calcitonin. Proc R Soc Lond B Biol Sci 170:71-80, 1968

47. Pearse AG: The cytochemistry and ultrastructure of polypeptide hormone-producing cells of the APUD series and the embryologic, physiologic and pathologic implications of the concept. J Histochem Cytochem 17:303-313, 1969

48. Pearse AGE: The diffuse neuroendocrine system: historical review. Front Horm Res 12:1-7, 1984

49. Reubi JC, Waser B, Khosla S, et al: In vitro and in vivo detection of somatostatin receptors in pheochromocytomas and paragangliomas. J Clin Endocrinol Metab 74:1082-1089, 1992

50. Rodriguez-Cuevas S, Lopez-Garza J, Labastida-Almendaro S: Carotid body tumors in inhabitants of altitudes higher than 2000 meters above sea level. Head Neck 20:374-378, 1998

51. Rosenwasser H: Carotid body tumor of the middle ear and mastoid. Arch Otolaryngol Head Neck Surg 41:64-67, 1945

52. Saldana MJ, Salem LE, Travezan R: High altitude hypoxia and chemodectomas. Hum Pathol 4:251-263, 1973

53. Salmenkivi K, Heikkila P, Liu J, et al: VEGF in 105 pheochromocytomas: enhanced expression correlates with malignant outcome. APMIS 111:458-464, 2003

54. Schmidt M, Fischer E, Dietlein M, et al: Clinical value of somatostatin receptor imaging in patients with suspected head and neck paragangliomas. Eur J Nucl Med Mol Imaging 29: 1571-1580, 2002

55. Schwaber MK, Glasscock ME, Nissen AJ, et al: Diagnosis and management of catecholamine secreting glomus tumors. Laryngoscope 94:1008-1015, 1984

56. Spector GJ, Ciralsky R, Maisel RH, et al: IV. Multiple glomus tumors in the head and neck. Laryngoscope 85:1066-1075, 1975

57. Spector GJ, Gado M, Ciralsky R, et al: Neurologic implications of glomus tumors in the head and neck. Laryngoscope 85: 1387-1395, 1975

58. Struycken PM, Cremers CW, Mariman EC, et al: Glomus tu- mours and genomic imprinting: influence of inheritance along the paternal or maternal line. Clin Otolaryngol 22:71-76, 1997

59. Takekoshi K, Ishii K, Shibuya S, et al: Stimulation of catecholamine biosynthesis via the protein kinase $\mathrm{C}$ pathway by endothelin-1 in PC12 rat pheochromocytoma cells. Biochem Pharmacol 63:977-984, 2002

60. Takekoshi K, Isobe K, Yashiro T, et al: Expression of vascular endothelial growth factor (VEGF) and its cognate receptors in human pheochromocytomas. Life Sci 74:863-871, 2004

61. Telischi FF, Bustillo A, Whiteman ML, et al: Octreotide scintigraphy for the detection of paragangliomas. Otolaryngol Head Neck Surg 122:358-362, 2000

62. Troughton RW, Fry D, Allison RS, et al: Depression, palpitations, and unilateral pulsatile tinnitus due to a dopamine-secreting glomus jugulare tumor. Am J Med 104:310-311, 1998

63. van der Mey AG, Maaswinkel-Mooy PD, Cornelisse CJ, et al: Genomic imprinting in hereditary glomus tumours: evidence for new genetic theory. Lancet 2:1291-1294, 1989

64. van Nederveen FH, Dannenberg H, Sleddens HF, et al: p53 alterations and their relationship to SDHD mutations in parasympathetic paragangliomas. Mod Pathol 16:849-856, 2003

65. Wang DG, Barros D'Sa AA, Johnston CF, et al: Oncogene expression in carotid body tumors. Cancer 77:2581-2587, 1996

66. Wang DG, Johnston CF, Barros D'Sa AA, et al: Expression of apoptosis-suppressing gene bcl-2 in human carotid body tumours. J Pathol 183:218-221, 1997

67. Wang DG, Johnston CF, Marley JJ, et al: Expression of the apoptosis-suppressing gene BCL-2 in pheochromocytoma is associated with the expression of C-MYC. J Clin Endocrinol Metab 82:1949-1952, 1997

68. Wasserman PG, Savargaonkar P: Paragangliomas: classification, pathology, and differential diagnosis. Otolaryngol Clin North Am 34:845-862, v-vi, 2001

69. Watanabe $\mathrm{K}$, Hiraki $\mathrm{H}$, Hasegawa $\mathrm{H}$, et al: Immunohistochemical localization of endothelin-1, endothelin-3 and endothelin receptors in human pheochromocytoma and paraganglioma. Pathol Int 47:540-546, 1997

70. Zielke A, Middeke M, Hoffmann S, et al: VEGF-mediated angiogenesis of human pheochromocytomas is associated to malignancy and inhibited by anti-VEGF antibodies in experimental tumors. Surgery 132:1056-1063, 2002

Manuscript received May 10, 2004.

Accepted in final form July 7, 2004.

Address reprint requests to: Jason Heth, M.D., Department of Neurosurgery, University of Michigan Department of Neurosurgery, Taubman Medical Center, Room 2128/0338, 1500 East Medical Center Drive, Ann Arbor, Michigan 48109. email: jheth@ umich.edu. 